\title{
Cocrystal Structure of the Redox-active Phenylenediamine and Quinonediimine Derivatives
}

\author{
Satoshi D. OHMUra,* Toshikazu Hirao,* Norikazu MiyoshI,** and Toshiyuki Moriuchi*广 \\ *Department of Applied Chemistry, Graduate School of Engineering, Osaka University, Yamada-oka, Suita, \\ Osaka 565-0871, Japan \\ **Department of Natural Sciences, Graduate School of Technology, Industrial and Social Sciences, \\ Tokushima University, 2-1 Minami-josanjima, Tokushima 770-0814, Japan
}

\begin{abstract}
The cocrystal structure of diethyl 2,5-bis\{[4-(ethoxycarbonyl)phenyl]amino\}terephthalate (PDA) and diethyl (3E,6E)-3,6bis $\{[4-($ ethoxycarbonyl)phenyl]amino $\}$ cyclohexa-1,4-diene-1,4-dicarboxylate (QDI) was determined by X-ray crystallography. The compound crystallizes in a triclinic system and was characterized thus: $P-1, a=8.6778(3) \AA, b=$ 13.0702(4) $\AA, c=13.5152(4) \AA, \alpha=79.1570(15)^{\circ}, \beta=71.8459(15)^{\circ}, \gamma=72.5962(16)^{\circ}, Z=1, V=1382.28(7) \AA^{3}$. The $R 1$ $[I>2 \sigma(I)]$ and $w R 2$ (all data) values are 0.078 and 0.190 , respectively, for all 4179 independent reflections. In the crystal, a polymeric alternating arrangement of PDA and QDI exists through a network of $\pi-\pi$ interactions, wherein both components adopt an anti-conformation of the $\pi$-conjugated backbones.
\end{abstract}

(Received July 24, 2019; Accepted August 23, 2019; Published on web November 10, 2019)

$\pi$-Conjugated organic molecules and polymers are promising candidates for a variety of applications, including flexible electrodes, ${ }^{1}$ light-emitting diodes, ${ }^{2}$ field-effect transistors ${ }^{3}$ and so on. Control of the redox properties of $\pi$-conjugated moieties can be used for further functionalization. Polyanilines is unique among the most common classes of conductive organic polymers and is used in many fields. ${ }^{4,5}$ Polyanilines consist of three different redox forms: fully reduced leucoemeraldine, semioxidized emeraldine and fully oxidized perniganiline base forms. Oligoanilines are used as model compounds of polyaniline, due to good solubility, well-defined structures and similar redox properties. Thereby, a series of oligoanilines have been synthesized and characterized. ${ }^{6-8}$ 1,4-Phenylenediamine and 1,4-quinonediimine are reduced and oxidized $\pi$-conjugated units of polyanilines, respectively. ${ }^{9}$ We previously demonstrated the redox switching of the emission properties of $\mathrm{Ru}(\mathrm{II})$ dipyridyl complexes bearing 1,4-phenylenediamine moieties by changing the redox states of the 1,4-phenylenediamine moieties. ${ }^{10-12}$ Moreover, switching and tuning of the luminescent properties of 2,5-bis(arylamino)terephthlate derivatives were also achieved. ${ }^{13-15}$ We herein report on a structural determination of cocrystal composed of diethyl 2,5-bis \{[4-(ethoxycarbonyl)phenyl]amino terephthalate (PDA) and diethyl (3E,6E)-3,6bis $\{[4-($ ethoxycarbonyl)phenyl]amino $\}$ cyclohexa-1,4-diene-1,4-

† To whom correspondence should be addressed.

E-mail: moriuchi@sci.osaka-cu.ac.jp

S. D. O. present address: Department of Natural Sciences, Graduate School of Technology, Industrial and Social Sciences, Tokushima University, 2-1 Minami-josanjima, Tokushima 770-0814, Japan. T. H. present address: The Institute of Scientific and Industrial Research, Osaka University, Mihoga-oka, Ibaraki, Osaka 567-0047, Japan.

T. M. present address: Division of Molecular Materials Science, Graduate School of Science, Osaka City University, 3-3-138 Sugimoto, Sumiyoshi, Osaka 558-8585, Japan. dicarboxylate (QDI) (Fig. 1).

In this research, the synthetic procedures of PDA and QDI have been already reported and fully characterized. ${ }^{13}$ The titled cocrystal was obtained by slow-evaporation of a solution of pure QDI in acetone under an air atmosphere. A red block crystal of suitable size was mounted on a grass fiber and all measurements were made on a Rigaku R-AXIS RAPID diffractometer using graphite-monochromated $\mathrm{Cu} K \alpha(\lambda=1.54187 \AA)$ radiation. ${ }^{16}$ The structure was solved by SIR2004, ${ }^{17}$ SHELXL Ver. 2018/3 ${ }^{18}$ and CrystalStructure Ver. 4.3 program. ${ }^{19}$ The refinement was carried out by using full-matrix least squares on $F^{2}$. $\mathrm{H}$ atoms involved in hydrogen-bonding interactions were located in difference electron-density maps and were fully refined. Crystal data, data collection, and structure refinement details are summarized in Table 1 , selected bond lengths $(\AA)$ and angles $\left({ }^{\circ}\right)$ are listed in Table 2 . The hydrogen-bonding geometry and

a)<smiles>CCOC(=O)c1ccc(Nc2cc(Nc3ccc(C(=O)OCC)cc3)c(C(=O)OCC)cc2NC)cc1</smiles>

b)<smiles>CCOC(=O)C1=CC(=Nc2ccc(C(=O)OCC)cc2)C(C(=O)OCC)=CC1=Nc1ccc(C(=O)OCC)cc1</smiles>

Fig. 1 (a) PDA and (b) QDI. Intramolecular hydrogen bonds are shown by the dotted line. 
Table 1 Crystal and experimental data

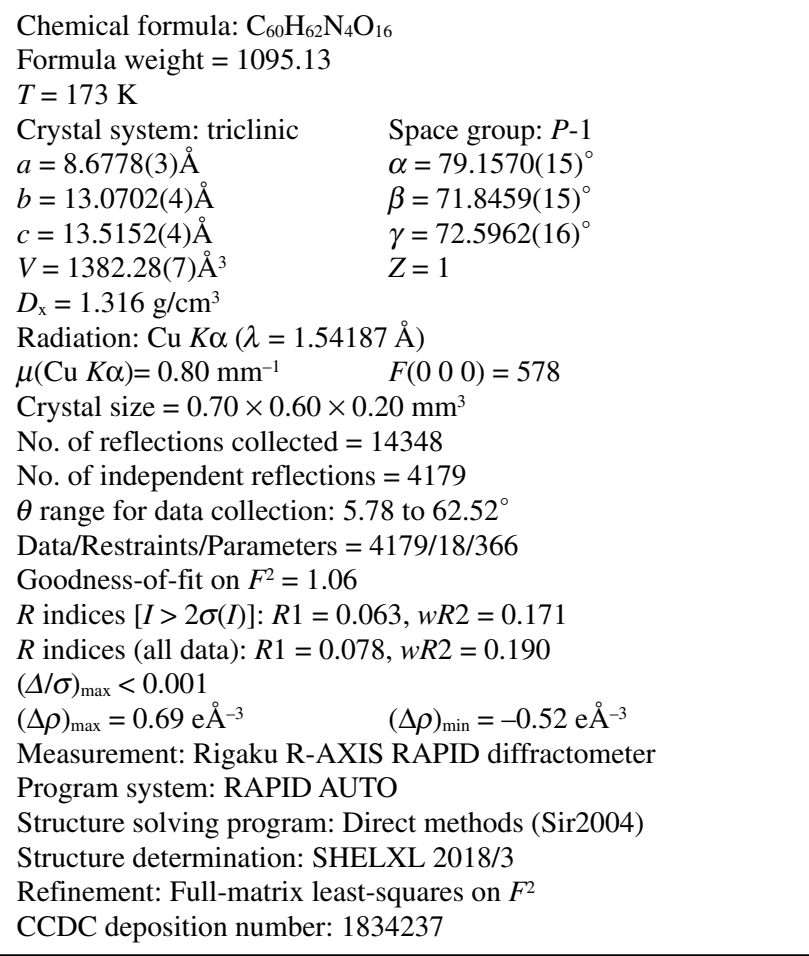

Table 2 Selected bond lengths $(\AA)$ and angles $\left({ }^{\circ}\right)$

\begin{tabular}{llll}
\hline $\mathrm{C} 2-\mathrm{C} 3 *$ & $1.389(4)$ & $\mathrm{C} 1-\mathrm{N} 1-\mathrm{C} 4$ & $127.4(2)$ \\
$\mathrm{C} 22-\mathrm{C} 23$ & $1.342(4)$ & $\mathrm{C} 21-\mathrm{N} 2-\mathrm{C} 24$ & $120.9(2)$ \\
$\mathrm{C} 1-\mathrm{N} 1$ & $1.394(3)$ & & \\
$\mathrm{C} 21-\mathrm{N} 2$ & $1.289(4)$ & & \\
\hline
\end{tabular}

Symmetry operators: $*,-x,-y+1,-z+1$.

selected dihedral angles between least-squares planes are given in Tables S1 and S2, respectively.

A perspective view of the obtained structure is shown in Fig. 2. The cocrystal has a 1:1 molar ratio of PDA and QDI, as shown. Therefore, some QDI might undergo a reduction to PDA under the conditions under which the cocrystal was obtained. The molecular structures of each component, PDA and QDI, and atomic numbering scheme are shown in Fig. 3. Both component molecules were found to form anticonformations of the $\pi$-conjugated moieties. In PDA component, intramolecular hydrogen bonds between the amino hydrogen of the phenylenediamine moiety and the carbonyl oxygen of the ethoxycarbonyl group at the central benzene ring were formed to induce anti-conformations of the $\pi$-conjugated phenylenediamine backbone (Fig. 3a). The terminal and central benzene rings are oriented with a dihedral angle of $47.4(1)^{\circ}$ (Table S2). This is probably due to a steric repulsion between the orthohydrogen atoms, despite the formation of intramolecular hydrogen bonds, which might induce a coplanar structure of the $\pi$-conjugated moiety. On the other hand, the QDI component has dihedral angles of $68.5(1)^{\circ}$ between the terminal and central benzene rings, as shown in Fig. $3 \mathrm{~b}$ and Table S2. This value is larger than that of the PDA component. The short imine bond length of QDI (1.29 A, Table 2) might cause a greater steric repulsion between the ortho-hydrogen atoms at the central and

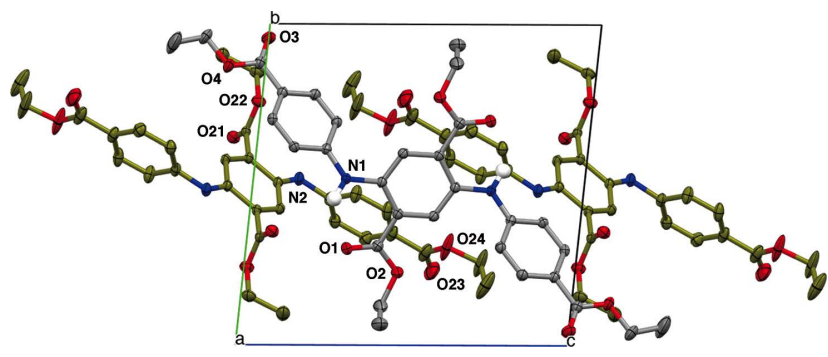

Fig. 2 View showing the crystal packing (gray, PDA; gold, QDI) down the $a$-axis. Displacement ellipsoids are drawn at the $30 \%$ probability level. C-bound $\mathrm{H}$ atoms are omitted for clarity.
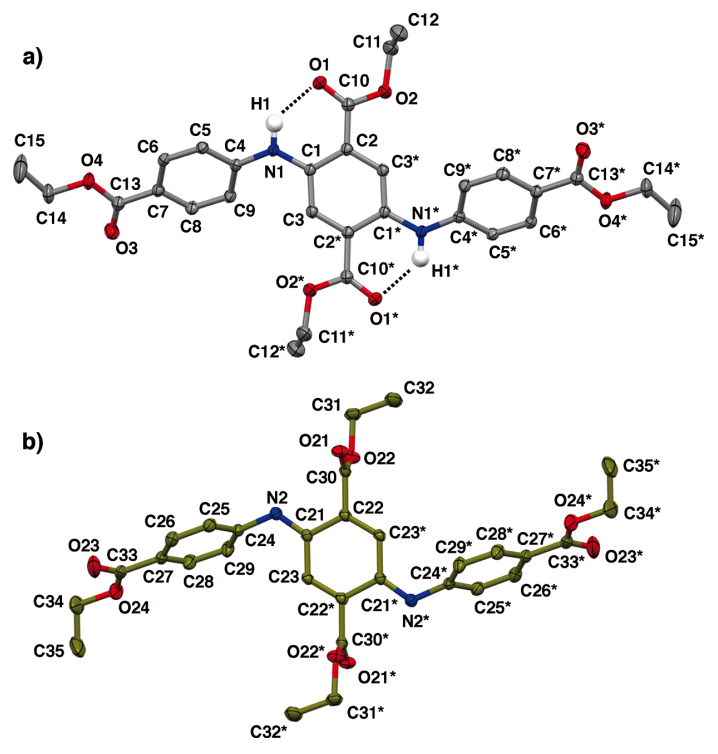

Fig. 3 Molecular structures of the (a) PDA and (b) QDI components, showing the atom-labelling scheme and the hydrogen bonds within the selected asymmetric unit (dashed lines). Displacement ellipsoids are drawn at the $30 \%$ probability level and the atoms marked with an asterisk (*) are at the symmetry position (a) $(-x,-y+1,-z+1)$ and (b) $(-x+1,-y+1,-z)$. C-bound $\mathrm{H}$ atoms are omitted for clarity.

terminal benzene rings. The $\pi-\pi$ interactions between the benzene rings of each component were observed, wherein central benzene ring (C21-C22-C23-C21*-C22*-C23*) of QDI was sandwiched between two terminal benzene rings (C4-C5C6-C7-C8-C9) of PDA (Fig. S1). The dihedral angle between the central benzene ring $\left(\mathrm{C} 21-\mathrm{C} 22-\mathrm{C} 23-\mathrm{C} 21^{*}-\mathrm{C} 22^{*}-\mathrm{C} 23 *\right)$ of QDI and the terminal benzene rings (C4-C5-C6-C7-C8-C9) of PDA was $17.2(2)^{\circ}$ with a distance of $3.72 \AA$ between their mean planes (Table S2). A polymeric alternating arrangement of PDA and QDI appeared to be stabilized through intermolecular interactions.

In conclusion, structural characterization of the cocrystal of diethyl 2,5-bis $\{[4-($ ethoxycarbonyl)phenyl $]$ amino $\}$ terephthalate (PDA) and diethyl (3E,6E)-3,6-bis \{[4-(ethoxycarbonyl)phenyl]amino cyclohexa-1,4-diene-1,4-dicarboxylate (QDI), which is an example of the cocrystal of the reduced and oxidized forms of $\pi$-conjugated units of polyanilines, was demonstrated. The single-crystal X-ray structure determination of the cocrystal reveals a polymeric alternating arrangement of PDA and QDI through a network of $\pi-\pi$ interactions, wherein both components 
adopt an anti-conformation of the $\pi$-conjugated backbones. The PDA component was found to form intramolecular hydrogen bonds between the amino hydrogen and carbonyl oxygen to induce the anti-conformation of the $\pi$-conjugated phenylenediamine backbone.

\section{Supporting Information}

This material is available free of charge on the Web at http:// www.jsac.or.jp/xraystruct/.

\section{References}

1. M. He, J. Jung, F. Qiu, and Z. Lin, J. Mater. Chem., 2012, 22, 24254.

2. L. Maggini, and D. Bonifazi, Chem. Soc. Rev., 2012, 41, 211.

3. N. E. Persson, P.-H. Chu, M. McBride, M. Grover, and E. Reichmanis, Acc. Chem. Res., 2017, 50, 932.

4. Z. Tian, H. Yu, L. Wang, M. Saleem, F. Ren, P. Ren, Y. Chen, R. Sun, Y. Sun, and L. Huang, RSC Adv., 2014, 4, 28195.

5. T. Sen, S. Mishra, and N. G. Shimpi, RSC Adv., 2016, 6 , 42196.

6. F.-L. Lu, F. Wudl, M. Nowak, and A. J. Heeger, J. Am.
Chem. Soc., 1986, 108, 8311.

7. R. A. Singer, J. P. Sadighi, and S. L. Buchwald, J. Am. Chem. Soc., 1998, 120, 213.

8. Y. Zhang, P. Straynowicz, and J. Christffers, Eur. J. Org. Chem., 2008, 3488.

9. W. R. Salaneck, D. T. Clark, and E. J. Samuelsen (ed.), "Science and Application of Conductive Polymers", 1991, Adam Hilger, New York.

10. T. Hirao and K. Iida, Chem. Commun., 2001, 431.

11. X. Shen, T. Moriuchi, and T. Hirao, Tetrahedron Lett., 2003, 44, 7711.

12. T. Moriuchi, J. Shiori, and T. Hirao, Tetrahedron Lett., 2007, 48, 5970.

13. S. D. Ohmura, T. Moriuchi, and T. Hirao, Tetrahedron Lett., 2010, 51, 3190.

14. T. Moriuchi, N. Kikushima-Honda, S. D. Ohmura, and T. Hirao, Tetrahedron Lett., 2010, 51, 4530.

15. T. Moriuchi, S. D. Ohmura, K. Morita, and T. Hirao, Chem. Asian. J., 2011, 6, 3206.

16. Rigaku RAPID-AUTO, Rigaku Corporation, Tokyo, Japan.

17. M. C. Burla, R. Caliandro, M. Camalli, B. Carrozzini, G. L. Cascarano, L. De Caro, C. Giacovazzo, G. Polidori, and R. Spagna, J. Appl. Cryst., 2005, 38, 381.

18. G. M. Sheldrick, Acta. Cryst. C71, 2015b, 3.

19. Rigaku, CrystalStructure. Ver 4.3, Rigaku Corporation, 2018, Tokyo, Japan. 